\title{
On-Chip Pixel Binning in Photon-Counting EMCCD-Based Gamma Camera: A Powerful Tool for Noise Reduction
}

\author{
Albert H. Westra, Jan W. T. Heemskerk, Student Member, IEEE, Marc A. N. Korevaar, Student Member, IEEE,
} Albert J. P. Theuwissen, Fellow, IEEE, Rob Kreuger, Kees M. Ligtvoet, and Freek J. Beekman, Senior Member, IEEE

\begin{abstract}
Charge Coupled Devices (CCDs) optically coupled to scintillation crystals can be used to construct high resolution gamma cameras. Previously, several groups have reported intrinsic detector spatial resolutions below 100 microns. When the CCD images can be read out fast enough, the energy and interaction position of individual gamma quanta can be estimated by a real-time image analysis of the scintillation light flashes. The Electron-Multiplying CCD (EMCCD) is well-suited for fast read out, since even at high frame rates it has extremely low readout noise. However, due to the often very low light levels present in scintillation gamma cameras, further reduction of noise is desirable. Here, the EMCCD is optically coupled to a $1000-\mu \mathrm{m}$-thick columnar CsI(TI) crystal for Tc-99m and I-125 imaging. We investigate noise reduction through summing of signals in pixels before the gain register and readout circuit of the EMCCD ("pixel binning"). We compare the energy and spatial resolution, photopeak efficiency (PE) and signal-to-noise ratio (SNR) of an EMCCD-based gamma camera for the case of binning vs. non-binning. When pixels were read out simultaneously in groups of 4 the spatial resolution is slightly worse in the direction of binning, but the number of false-positive counts resulting from background noise for I-125 was reduced by $74 \%$ compared to the no binning case. We conclude that the use of charge binning when reading out EMCCDs may significantly improve the energy spectra and noise properties of CCD-based high-resolution gamma cameras.
\end{abstract}

Index Terms-Binning, electronics, EM-CCD, gamma camera, SPECT.

Manuscript received September 25, 2007; revised April 09, 2008. Current version published October 07, 2009. This work was supported in part by the Netherlands Organization for Scientific Research (NWO) under Grant 917.36. 335 .

A. H. Westra and K. M. Ligtvoet are with the Department of Medical Technology and Clinical Physics, University Medical Center, Utrecht, The Netherlands.

J. W. T. Heemskerk and M.A.N. Korevaar are with the Image Sciences Institute, University Medical Center, Utrecht, The Netherlands and also with the Section Radiation Detection and Medical Imaging, Department of Radiation, Radionuclides and Reactors, Delft University of Technology, Mekelweg 15, 2629JB, Delft, The Netherlands (e-mail: j.w.t.heemskerk@tudelft.nl).

A. J. P. Theuwissen is with the Faculty of Electrical Engineering, Delft University of Technology, Delft, The Netherlands.

R. Kreuger is with the Section Radiation Detection and Medical Imaging, Department of Radiation, Radionuclides and Reactors, Delft University of Technology, Delft, The Netherlands.

F. J. Beekman is with the Image Sciences Institute, University Medical Center, Utrecht, The Netherlands; the Section Radiation Detection and Medical Imaging, Department of Radiation, Radionuclides and Reactors, Delft University of Technology, Delft, The Netherlands; and also with MILABS, Utrecht, The Netherlands.

Digital Object Identifier 10.1109/TNS.2009.2012931

\section{INTRODUCTION}

$\mathbf{H}$ IGH-RESOLUTION gamma and X-ray detectors are currently under development in a large number of laboratories (e.g., [1]-[15]). Such detectors can be useful to improve small-animal SPECT systems [8], [12], [16]-[18]. Very high spatial resolution combined with energy discrimination capabilities can be obtained through the use of micro-columnar scintillation crystals that are read out by EMCCDs (Fig. 1, [10], [12], [14]). These compact gamma cameras can determine the position and energy of individual scintillation events (photoncounting) and in some cases achieve spatial resolutions better than $60 \mu \mathrm{m}$ [10], [12], [14], [15]. The use of back-illuminated instead of front-illuminated EMCCDs leads to significant improvements with respect to energy resolution and the number of false-positive counts [19].

Despite the continuous improvement of the spatial and energy resolution and noise properties of EMCCD-based gamma cameras, additional noise reduction can widen the range of applications.

In low light level applications, the performance of an EMCCD is limited by the thermally generated dark current and the clock-induced charge (CIC) that is the result of the charge transfer through the device([21], [22]). The dark current can be suppressed by cooling the EMCCD; CIC however is a temperature-independent noise source.

The amount of CIC noise is proportional to the number of transfers that the signal is subject to before readout. Here we reduce this number of transfers by summing the signal of multiple lines (binning) before it is transferred through the serial readout and gain register. Binning has been applied in other CCD-based gamma cameras ([14], [15]) for increasing frame rates. The goal of the present paper is to deduce the efficacy of binning for reducing the CIC noise. To this end, we present energy spectra, SNR measurements as well as image profiles for spatial resolution measurements obtained with and without binning.

\section{Materials AND MethodS}

\section{A. EMCCD, Scintillators and Optic Coupling}

An Electron-Multiplying CCD (EMCCD) is a CCD with an added feature: internal gain in the charge domain (i.e., before readout). Because of this internal gain an EMCCD is less sensitive to readout noise, even at high readout speeds (e.g., several 
a

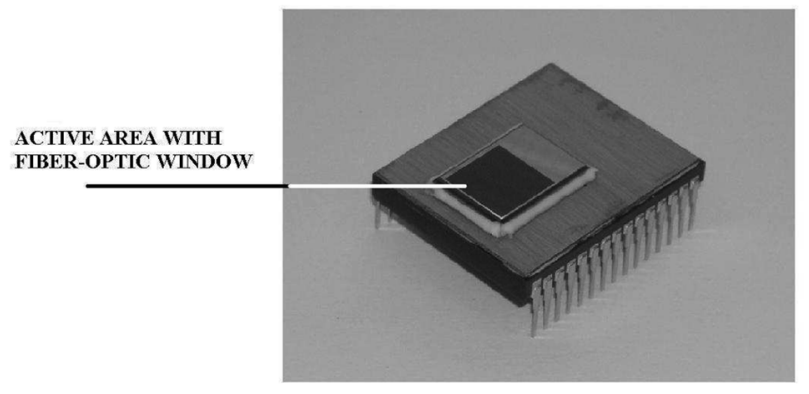

b

COLUMNAR SCINTILLATOR

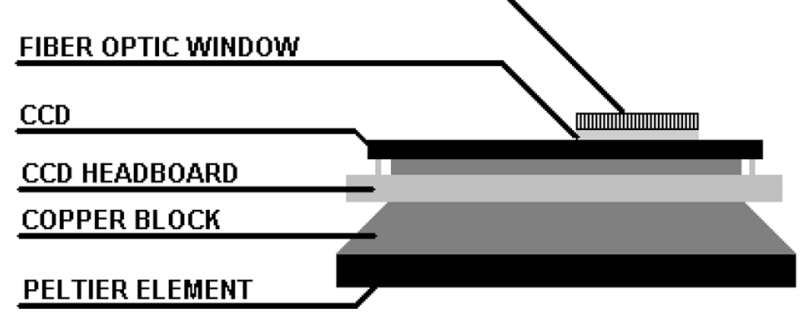

Fig. 1. EMCCD chip with fiber-optic window (a) and a compact EMCCDbased gamma camera (b). A Peltier element cools the CCD chip, thereby reducing the dark current noise.

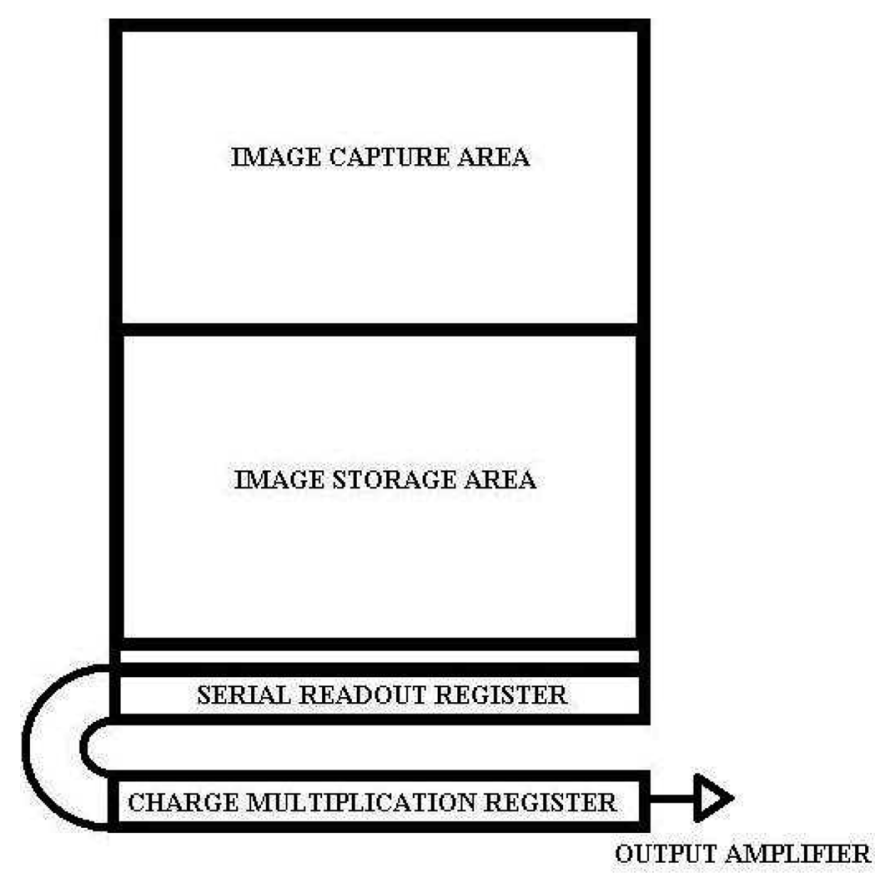

Fig. 2. Schematic lay-out of the EMCCD. The internal electron multiplication gain is achieved in the charge multiplier that has been added before the readout capacitor. The binning of lines is performed by summing of the signal of multiple lines in the serial readout register. This is achieved through downward transfer of lines into the register, before the previous line(s) has been shifted serially through the register. The signal of any number of lines can thus be summed before it enters the charge multiplier [20].

Mpixels/second). The internal gain is provided by electron multiplication (avalanche multiplication or impact ionization) in the gain register (see Fig. 2). Details of the EMCCD technology are available for example in [23]-[26].

The EMCCD used in the present work is the back-illuminated CCD97 from E2V Technologies, which has 512 lines of 512 pixels, with a pixel size of $16 \times 16 \mu \mathrm{m}^{2}$ and a quantum efficiency exceeding $90 \%$ for visible light in the range of 500 to $650 \mathrm{~nm}$ (Fig. 1(a), [19], [26]). It is cooled to $-50^{\circ} \mathrm{C}$ to reduce the dark current to levels below $2 \cdot 10^{-2} \mathrm{e}^{-}$/pixel/frame (according to the manufacturer). The temperature-independent CIC noise, which is also called spurious charge or charge transfer noise, has a level of approx. $3.3 \cdot 10^{-5} \mathrm{e}^{-/ \text {transfer }}$ for our read-out frequency of 50 frames per second at a pixel frequency of $11 \mathrm{MHz}$ [22], [26]. In the worst case scenario, the charge has to pass through 512 lines of the image area, 520 lines of the storage area and 552 serial transfer columns before the gain stage, adding up to $5.2 \cdot 10^{-2} \mathrm{e}^{-/ \text {pixel. It is therefore }}$ the dominant noise source in our application.

For the conversion of the incident gamma rays to visible photons, we used a $1000-\mu \mathrm{m}$-thick micro-columnar $\mathrm{CsI}(\mathrm{Tl})$ scintillation crystal (Hamamatsu FOS, type J6671), which has a capture efficiency of almost $100 \%$ for I- 125 and $\sim 30 \%$ for $\mathrm{Tc}-99 \mathrm{~m}$. The peak wavelength of the emission spectrum lies at $550 \mathrm{~nm}$, which is in the optimal range of our EMCCD, while the columnar structure of $\mathrm{CsI}(\mathrm{Tl})$ yields a high spatial resolution. A fiber-optic taper can be used to increase the effective detector area per CCD, but in our experiment a 3-mm-thick straight fiber optic window is present for protection of the CCD. The scintillation crystal was coupled to the fiber-optic window using Bicron BC-630 silicon optical grease.

\section{B. Photon-Counting Algorithm}

The electronics board that drives and reads out the EMCCD is connected to a Digital Signal Processor (DSP), type TMS320C6416 from Texas Instruments. This DSP executes a photon-counting algorithm that performs a real-time analysis of each of the frames of the EMCCD to detect and localize individual scintillation events.

Summarized, the algorithm proceeds as follows: first, before each measurement sequence an average (dark) background image is acquired. Then for each frame during acquisition, this background image is subtracted from the raw EMCCD image. Next, a filtering step is performed by convolution of the background-corrected image with a 2D Gaussian filter. In this filtered image scintillation events will appear as bright regions and the peak position and intensity of each of these bright regions are transferred in list mode to a PC. The peak amplitude of the bright spots after filtering provides a measure for the energy that has been released in the scintillation event.

A more detailed description of the algorithm can be found in [10]; a description of the influence of the width of the 2D Gaussian kernel on the spatial and energy resolution of the gamma camera can be found in [19]. The physical width of the Gaussian convolution $(\sigma)$ in both the horizontal and vertical direction is set to the same value (i.e., $\sigma$ is $64 \mu \mathrm{m}$ ), for both the case of binning and not binning. 


\section{On-Chip Binning}

To improve the noise characteristics of our EMCCD, we have binned the signal of multiple lines before their transfer through the serial readout and gain register. This effectively reduces the number of transfers that the signal is subject to, which in turn reduces the amount of CIC noise since it is linearly dependent on the number of transfers. Binning also reduces the susceptibility of the signal to the noise in the serial readout and gain register and readout circuit.

In normal operation EMCCDs are read out the following way: after line-by-line (i.e., parallel) transfer of the signal from the storage area to the serial readout register, it is shifted pixel by pixel (i.e., serially) through the gain stage and the readout capacitor. Due to the fact that the readout electronics of our camera have been developed in-house, we have the ability to transfer multiple lines (rows) into the serial register before the serial transfer (binning). Effectively we thus sum the charge signal of multiple pixels in a column before it is transferred through the gain stage. Unfortunately, due to the structure of the device, binning of the charge in the direction of the rows can only be performed just before the read-out capacitor ${ }^{1}$. Though this can reduce the influence of the read-out noise it will have no effect on the CIC noise. Consequently, we only perform vertical binning in our comparison.

About half of the total number of transfers that the signal is subject to is in the serial readout and gain register; therefore binning could reduce the influence of the CIC by almost half. For instance, binning 4 lines would reduce the contribution of the CIC noise for the serial transfers through the readout and gain register, compared to the signal, by a factor of 4 ; instead of shifting 4 pixels through the registers, we are shifting only 1. Since the serial and gain register transfers amount to half the total number of transfers, this effectively reduces the total CIC noise by $\sim 40 \%$. Furthermore, summation of the signal of multiple pixels increases the signal in the serial register. The larger signal is less susceptible to the noise added in the readout circuit and to the standard deviation of the total noise ${ }^{2}$, thereby increasing the signal to noise ratio further. However, the contribution of the statistical noise generated in the gain register will not improve. The stochastic nature of the electron multiplication gain leads to a factor $\sqrt{ } 2$ increase of the variation of the noise over the gain register, independent of the amount of charge entering the gain stage.

Since binning occurs in the charge domain it is an essentially noiseless operation. It is often used in high-speed microscopy or fluoroscopy to either improve the readout rate of the camera or to reduce the influence of the noise in the readout capacitor [27]. However, binning will often lead to loss of spatial resolution as the pixel size is increased by the binning factor.

\section{Measurements}

To determine the spatial and energy resolution, photopeak efficiency (PE) and signal-to-noise ratio (SNR) of the camera, line

\footnotetext{
${ }^{1}$ Furthermore, in our particular setup horizontal binning can only be applied in conjunction with vertical binning, while vertical binning can also be applied independently.

${ }^{2}$ Note, however, that this latter effect could also be achieved by summing or averaging the signal after read-out.
}

pattern images were acquired using a setup with two (4 $\mathrm{mm}$ thick) tungsten blocks separated by a narrow slit (30 $\mu \mathrm{m}$ wide). Tc-99m (141 keV) and I-125 (27-35 keV) sources are placed behind the slit and a line pattern is projected through the slit onto the scintillator. The use of a slit to determine the line spread function is a standard method for measuring intrinsic resolution of clinical gamma cameras.

Energy spectra were recorded for the I-125 and Tc-99m sources consecutively for the irradiated area of the CCD (50 lines wide centered on the image of the slit). The energy resolution is determined from the FWHM of the peaks of the energy spectra after subtraction of the background level.

The spatial resolution is defined as the Full Width at Half Maximum (FWHM) of the line spread function corrected for the width of the gamma beam of $30 \mu \mathrm{m}$, on the assumption that both the beam itself and the response from an infinitely narrow beam are Gaussian. The scintillation events that are accepted for the determination of the spatial resolution are those with a light yield that fall within a $10 \%$ energy window $( \pm 14 \mathrm{keV})$ symmetrical around the photo-peak energy for Tc-99m and within a window ranging from $20 \mathrm{keV}$ to $50 \mathrm{keV}$ for I-125.

The photopeak efficiency (PE) was determined as the ratio of the signal counts within the photopeak compared to the background counts. For both the I-125 and Tc-99m photopeak (of the independently measured energy spectra), counts that lie below the baseline of the photopeak, were identified as background; counts above the baseline were identified as photopeak signal (Fig. 3). The signal-to-noise ratio (SNR) is defined as the total number of counts in the energy windows (I-125: 20 to $50 \mathrm{keV}$; Tc-99m: 126 to $155 \mathrm{keV}$ ) divided by the number of noise-induced false-positive counts detected in the appropriate energy windows of dark (unirradiated) images. The count rate of the experimental setup (several $\mathrm{cps} / \mathrm{mm}^{2}$ below the slit) is similar to what can be expected in practical small-animal pinhole SPECT imaging.

We compare measurements performed with binning of 2 and 4 lines with no binning. For this comparison the gain of the EMCCD was adjusted such that the output signal of the camera was equal for all cases. The EMCCD was irradiated with the slit oriented in both the horizontal and vertical direction (on the EMCCD image) in order to determine resp. the spatial resolution both along the direction of binning (i.e., the vertical direction) and perpendicular to the direction of binning (horizontal). Each of the measurements described below consists of a total number of 25000 analyzed frames.

\section{RESULTS}

\section{A. Energy Resolution, PE and SNR}

Fig. 4 shows the energy spectra acquired with I-125 and Tc-99m for the case without binning and for binning of 2 and 4 lines. Furthermore, the background spectra measured without any radioactive sources present are also provided.

The scale of the energy axis is calibrated assuming that the peaks of I-125 and Tc-99m are situated at $30 \mathrm{keV}$ and $141 \mathrm{keV}$ respectively. The energy resolution is determined as the FWHM of the separate I-125 and Tc-99m peaks after subtraction of the 

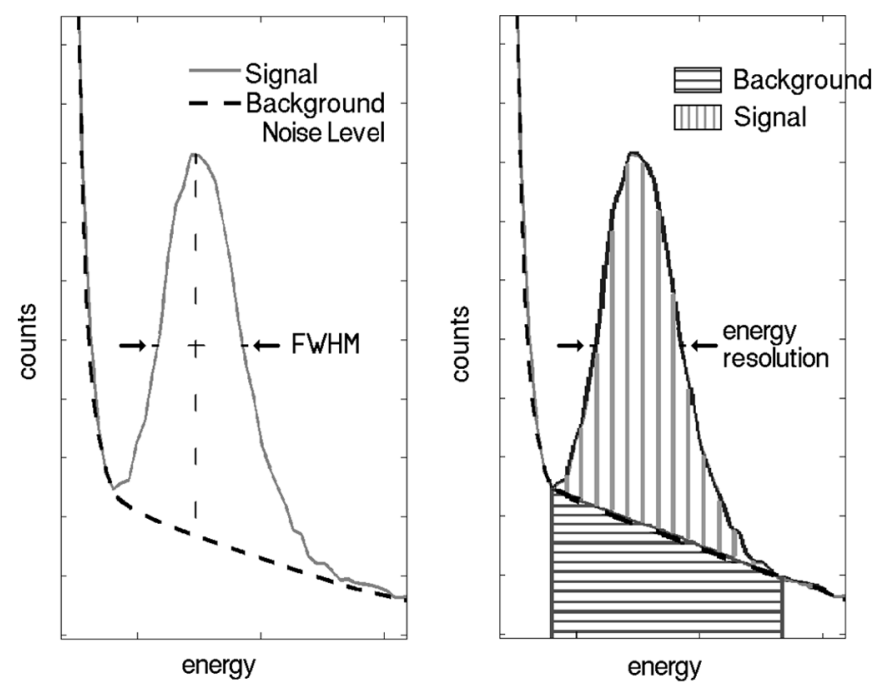

Fig. 3. Definition of the energy resolution and photopeak efficiency (PE) deduced from the energy spectra. The energy resolution is determined as the FWHM of the photopeak after subtraction of the background noise level. For determination of the photopeak efficiency the number of counts above the background level is considered signal; the number of counts below this level is considered background (right).

background noise level. The I-125 peaks could be well fitted with Gaussians, the Tc-99m peaks less so.

The energy resolution was estimated to be $32 \%$ FWHM (45 keV) for Tc-99m and 59\% (17.6 keV) for I-125 without binning. Binning of 4 lines resulted in an energy resolution of $28 \%$ (40 keV) for Tc-99m and $53 \%(15.9 \mathrm{keV})$ for $\mathrm{I}-125$, showing an improvement for both. The energy resolutions both with and without binning are listed in Table I.

The PE measurements with and without binning, for both the I- 125 and Tc-99m energy windows, are presented in Fig. 5 and Tables II and III. The signal is determined from the energy plots (Fig. 5) as the vertically striped area under the peak: the background is determined from the horizontally striped background level. Binning improved the PE by $118 \%$ for I- 125 and by $58 \%$ for Tc- $99 \mathrm{~m}$. Tables II and III also include the ratio of the total signal of the photopeaks to the false positive counts in identical energy windows for completely dark (unirradiated images). For the I-125 energy window these numbers indicate a clear improvement of the SNR; for the Tc-99m window the SNR does not seem to improve. For I-125 an important parameter, the number of false counts in the energy windows for the unirradiated $\mathrm{CCD}$, decreases significantly with binning.

\section{B. Spatial Resolution Measurements}

Fig. 6 shows I-125 image profiles taken perpendicular to the line pattern without binning (single line readout) and for binning of 4 lines. Fig. 6 left shows the profile when the line is projected vertically (in the EMCCD image); Fig. 6 right shows the profile when the line is projected horizontally. In the first case the profile of the line lies perpendicular to the direction in which the binning takes place, and thus the effective pixel size does not change with binning. In the latter case, the binning of lines is along the profile of the projected line, and thus the effective pixel size for binning 4 lines is $64 \mu \mathrm{m}$ in that direction.
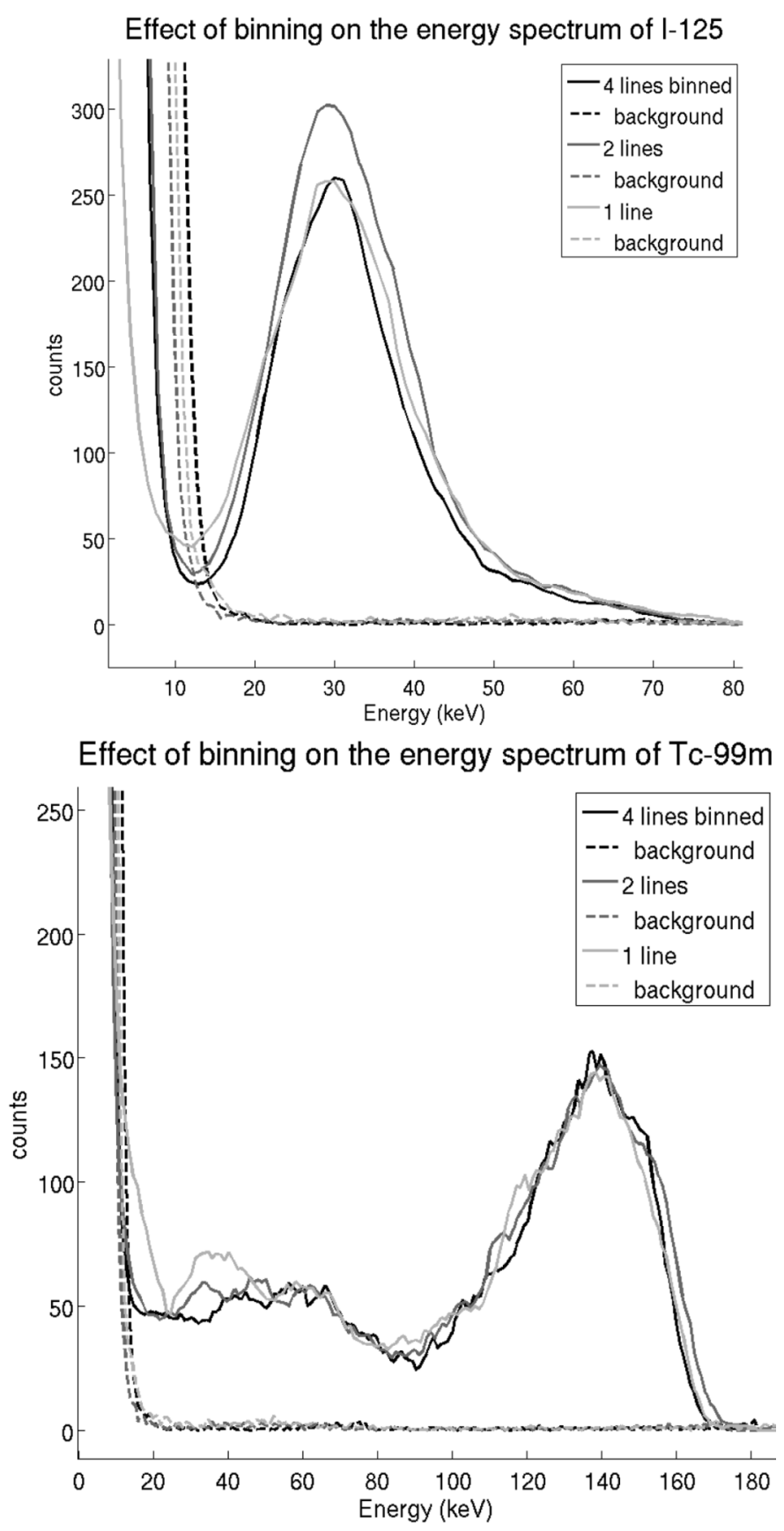

Fig. 4. Comparison of the energy spectra, when imaging I-125 and Tc-99m separately, for the cases of binning of lines vs. no binning. The spectra have been measured for an area of 50 lines wide centered on the image of the slit; the non-irradiated background spectra have been measured for the entire CCD.

The resolutions determined from Full Width at Half Maximum (FWHM) of the profiles for both Tc-99m and I-125 are listed in Table I. The uncertainty on the spatial resolution measurements is on the order of $1.5 \mu \mathrm{m}$. Use of binning slightly improves the spatial resolution in the direction perpendicular to the binning of lines for Tc-99m from $55.8 \mu \mathrm{m}$ to $53.5 \mu \mathrm{m}$ and for I-125 from $46.1 \mu \mathrm{m}$ to $42.4 \mu \mathrm{m}$. The loss of resolution in the parallel direction, along which binning occurs, is from $54.0 \mu \mathrm{m}$ to $83.8 \mu \mathrm{m}$ for Tc-99m and from $49.7 \mu \mathrm{m}$ to $76.7 \mu \mathrm{m}$ for I- 125 . This is far less than the effective increase in pixel size. The spatial resolution in the parallel direction is still better than 1.5 pixel lengths $(96 \mu \mathrm{m})$. 


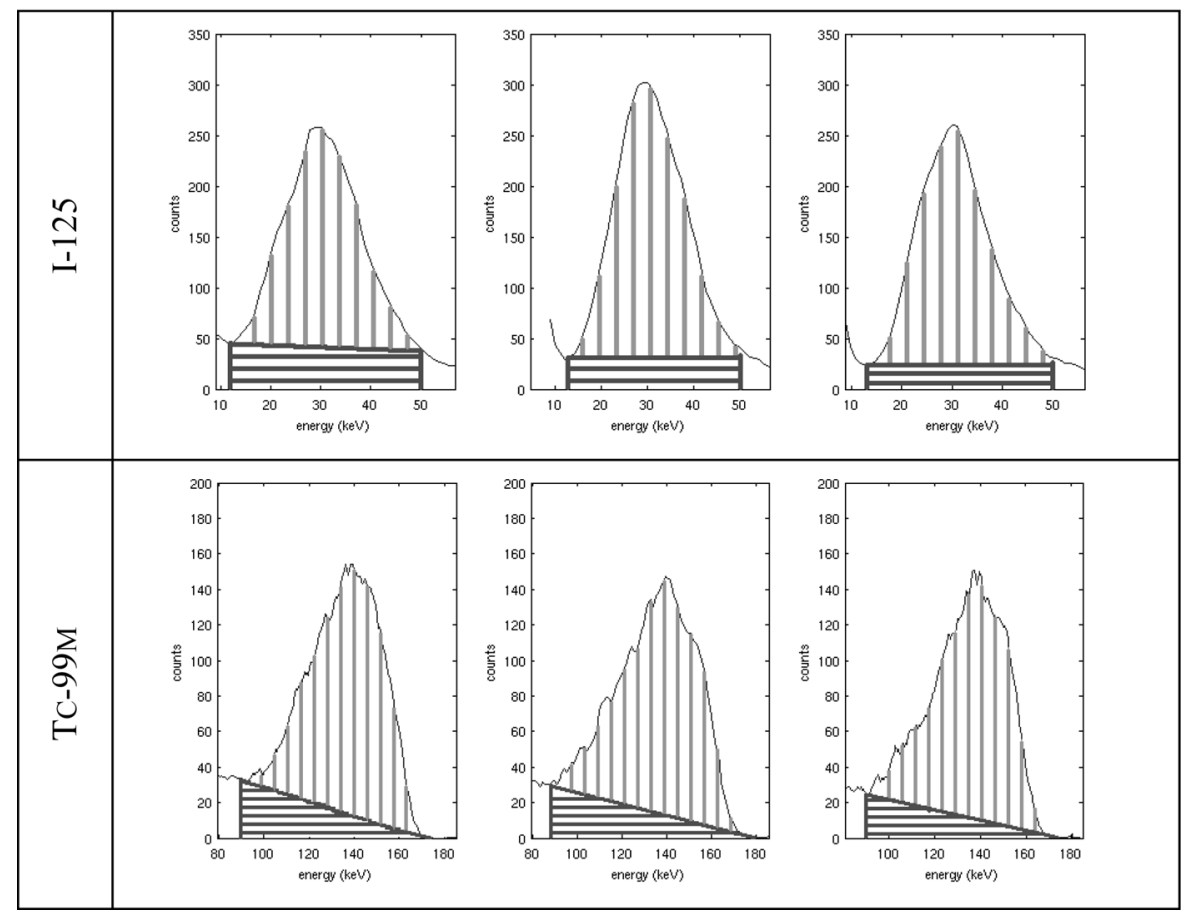

Fig. 5. Comparison of the photopeak efficiency of I-125 (top) and Tc-99m (bottom) for the case of binning of lines vs. no binning. These are derived from the individual spectra of both sources. The horizontally delineated areas mark the background counts, the vertically delineated areas the signal counts.

TABLE I

ENERGY AND SPATIAL RESOLUTION FOR TC-99M AND I-125. SUMMARY OF THE RESOLUTION MEASUREMENT RESUlts, AS MEASURED From THE ENERGY SPECTRA (FIG. 4) AND THE CORRESPONDING TC-99M AND I-125 IMAGE PROFILES (FIG. 7), FOR No BINNING AND FOR BINNING 2 AND 4 LINES

\begin{tabular}{cccccc}
\hline & & \multicolumn{2}{c}{ Tc-99m } & \multicolumn{2}{c}{ I-125 } \\
\cline { 3 - 6 } & binning & horizontal & vertical & horizontal & vertical \\
\hline Spatial & 1 & 55.8 & 54.0 & 46.1 & 49.7 \\
Resolution & 2 & 50.9 & 60.0 & 42.4 & 48.0 \\
$(\mu \mathrm{m})$ & 4 & 53.5 & 83.8 & 42.4 & 76.7 \\
\hline \multirow{2}{*}{ Energy } & 1 & {$[\mathrm{keV}]$} & {$[\%]$} & {$[\mathrm{keV}]$} & {$[\%]$} \\
resolution & 2 & 45 & 32 & 17.6 & 58.7 \\
& 4 & 46 & 32.6 & 17.5 & 58.3 \\
& & 40 & 28.4 & 15.9 & 53.0 \\
\hline
\end{tabular}

\section{DISCUSSION}

In the present paper we have shown that the use of binning increases the PE and SNR and reduces noise induced false-positive counts in CCD-based gamma camera for imaging of I-125. Furthermore we showed that binning results in some improvement of the energy resolution, even when this has been determined after the subtraction of the background noise. Without this background correction the energy resolution for not binning would be far worse. Unfortunately the improvements in spectral characteristics and energy resolution come at the cost of some loss in spatial resolution in the direction of binning; increasing the effective pixel height by a factor of 4 degrades the resolution along the direction of binning by just over $50 \%$. However, the resulting spatial resolution is in the range of the effective pixel
TABLE II

SUMMARY OF THE PE AND SNR MEASUREMENTS FOR BINNING AND WITHOUT BINNING FOR I-125. SUMMARY OF THE PHOTOPEAK EFFICIENCY (PE) AND SNR RESUlts, AS MEASURED From tHE ENERGY SPECTRA (Fig. 5) AND THE CORRESPONDING ENERGY WINDOWS OF THE DARK IMAGES, FOR NO BINNING AND FOR BINNING 2 AND 4 LINES

\begin{tabular}{lcccc}
\hline & binning & $\begin{array}{c}\text { Energy window } \\
(\mathrm{keV})\end{array}$ & $\begin{array}{c}\text { Total peak area } \\
/ \text { background }\end{array}$ & $\begin{array}{c}\text { Photopeak } \\
\text { efficiency }\end{array}$ \\
\hline 50 lines only & $\begin{array}{c}\text { no } \\
\text { binning } \\
2 \text { lines }\end{array}$ & $12-50$ & $4950 / 1540$ & 2.2 \\
& 4 lines & & $5746 / 1110$ & 4.2 \\
& & & $5068 / 871$ & 4.8 \\
\hline \hline & binning & Energy window & Total counts/ & SNR \\
& & $(\mathrm{keV})$ & Noise counts & \\
\hline Line signal & no & $20-50$ & $4332 / 357$ & 12.1 \\
compared to & binning & & & 18.2 \\
dark image & 2 lines & & $5352 / 294$ & 50.4 \\
& 4 lines & & $4636 / 92$ & \\
\hline
\end{tabular}

size; we believe that for configurations where the spatial resolution is in the range of multiple times the pixel size or larger (e.g., in the case of thicker crystals) the loss of spatial resolution due to binning will be relatively small.

In the direction perpendicular to the binning, one would expect at least to see an improvement of the spatial resolution. For the I-125 signal indeed we see some improvement, 
TABLE III

SUMMARY OF THE PE AND SNR MEASUREMENTS FOR BINNING AND WITHOUT BINNING FOR TC-99M. SUMMARY OF THE PHOTOPEAK EFFICIENCY (PE) AND SNR Results, as MEASUREd From the ENERgy SPeCtra (Fig. 5) AND THE CORRESPONDING ENERGY WINDOWS OF THE DARK IMAGES, FOR NO BINNING AND FOR BINNING 2 AND 4 LINES

\begin{tabular}{|c|c|c|c|c|}
\hline & binning & $\begin{array}{c}\text { Energy window } \\
(\mathrm{keV})\end{array}$ & $\begin{array}{c}\text { Total peak area } \\
\text { / background }\end{array}$ & $\begin{array}{l}\text { Photopeak } \\
\text { efficiency }\end{array}$ \\
\hline \multirow[t]{4}{*}{50 lines only } & $\begin{array}{c}\text { no } \\
\text { binning }\end{array}$ & $90-180$ & $6735 / 1492$ & 3.5 \\
\hline & 2 lines & & $6487 / 1218$ & 4.3 \\
\hline & 4 lines & & $6610 / 1007$ & 5.6 \\
\hline & binning & $\begin{array}{c}\text { Energy window } \\
(\mathrm{keV})\end{array}$ & $\begin{array}{l}\text { Total counts / } \\
\text { Noise counts }\end{array}$ & SNR \\
\hline $\begin{array}{l}\text { Line signal } \\
\text { compared to }\end{array}$ & $\begin{array}{c}\text { no } \\
\text { binning }\end{array}$ & $126-154$ & $3722 / 75$ & 49.6 \\
\hline \multirow[t]{2}{*}{ dark image } & 2 lines & & $3515 / 74$ & 47.5 \\
\hline & 4 lines & & $3909 / 80$ & 48.9 \\
\hline
\end{tabular}

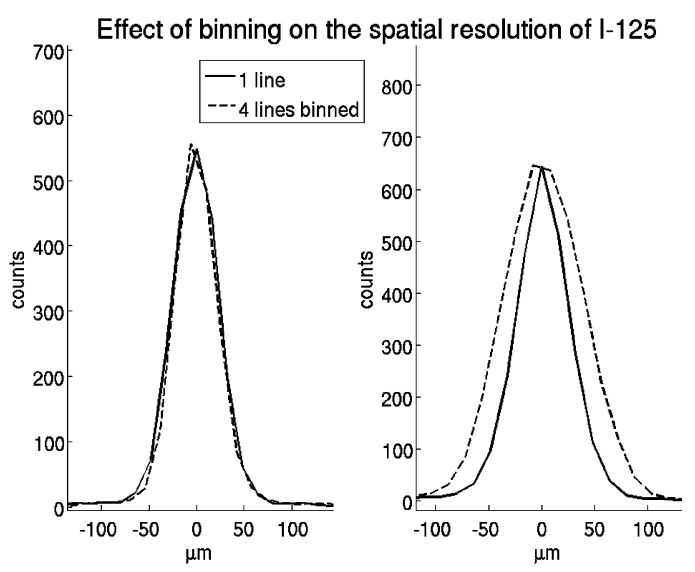

Fig. 6. Image profiles of the projection of the slit in the I-125 energy window, not binned (1 line) and binned for 4 lines. The left figure shows the effect of binning on the resolution in the horizontal direction; the right figure shows the effect of binning on the resolution in the vertical direction. In the vertical direction binning increases the effective pixel size by a factor of 4 to $64 \mu \mathrm{m}$.

for the Tc-99m signal an improvement cannot be detected, which can be explained by the strong signal in the case of $140 \mathrm{keV}$ photons.

We expect that particularly in case of binning more pixels, further improvements in energy and spatial resolution are possible with the application of advanced centroiding algorithms. In the direction of binning, the current spatial resolution is less than 1.5 times the pixel size; this might very well be reduced by e.g., Anger logic or fitting algorithms that enable sub-pixel determination of the location of the individual scintillation events. Recently, a Maximum Likelihood Estimation Maximization (MLEM) algorithm has been developed for 3D position and energy estimation that can partly correct for depth-of-interaction (DOI) effects [28]. At this stage however, such algorithms are computationally intensive.
Even though sufficient energy resolution is essential in clinical SPECT in order to distinguish primary photons from scattered photons, small-animal SPECT results in relatively small numbers of scattered photons. This is due to the small sizes of the objects being imaged. Furthermore, the amount of scatter in pinhole apertures is quite low, and does not give rise to strong contamination of projection data even without energy discrimination [29]. Therefore the energy discrimination capabilities of our prototype gamma camera should prove more than sufficient for application in small-animal pinhole SPECT. The optimal tradeoff between spatial resolution and energy resolution and noise depends on the specific imaging task. For example for very small objects (e.g., mice) scatter rejection is of minor importance and spatial resolution can be maximized. For clinical applications a lower intrinsic resolution is required while energy resolution can be very important.

\section{CONCLUSION}

In the present work we have investigated energy and spatial resolution and spectral characteristics of an EMCCD-based gamma camera, when the signal of multiple pixels in a column is summed before entering the gain register of the EMCCD. The use of this pixel binning leads to improvements in energy resolution, while maintaining sufficient spatial resolution. Further improvements might be possible if the signal could be binned for columns as well as for lines.

The signal-to-noise ratio has improved significantly for the low-energy I-125 $\gamma$-photons. Furthermore binning can facilitate the readout of the EMCCD at higher frame rates. We conclude that binning in EMCCDs is a promising technique, in particular for low light level detection, such as in gamma cameras used for SPECT imaging.

\section{ACKNOWLEDGMENT}

A. W. and J. H. share first authorship of this paper.

\section{REFERENCES}

[1] J. L. Matteson, W. Coburn, F. Duttweiler, W. A. Heindl, G. L. Huszar, P. C. Leblanc, M. R. Pelling, L. E. Peterson, R. E. Rothschild, R. T. Skelton, P. L. Hink, and C. Crabtree, "CdZnTe arrays for astrophysics applications," Proc. SPIE, vol. 3115, pp. 160-175, 1997.

[2] H. B. Barber, "Applications of semiconductor detectors to nuclear medicine," Nucl. Instrum. Methods Phys. Res. A, vol. A436, pp. 102-110, 1999.

[3] L. Menard, Y. Charon, M. Solal, P. Laniece, R. Mastrippolito, L. Pinot, L. Ploux, M. Ricard, and L. Valentin, "POCI: A compact high resolution gamma camera for intra-operative surgical use," IEEE Trans. Nucl. Sci., vol. 45, no. 3, pp. 1293-1297, Jun. 1998.

[4] Z. He, W. Li, G. F. Knoll, D. K. Wehe, J. Berry, and C. M. Stahle, "3-D position sensitive CdZnTe gamma-ray spectrometers," Nucl. Instrum. Methods Phys. Res. A, vol. A422, pp. 173-178, 1999.

[5] D. Vavrik, J. Jakubek, J. Visschers, S. Pospisil, C. Ponchut, and J. Zemankova, "First tests of a medipix-1 pixel detector for X-ray dynamic defectoscopy," Nucl. Instrum. Methods Phys. Res. A, vol. A487, no. 1-2, pp. 216-223, 2002.

[6] C. Fiorini, A. Longoni, F. Perotti, C. Labanti, E. Rossi, P. Lechner, H. Soltau, and L. Struder, "A monolithic array of silicon drift detectors coupled to a single scintillator for gamma-ray imaging with sub-millimeter position resolution," Nucl. Instrum. Methods Phys. Res. A, vol. A512, pp. 265-271, 2003.

[7] J. E. Lees, G. W. Fraser, A. Keay, D. Bassford, R. Ott, and W. Ryder, "The high resolution gamma imager (HRGI): A CCD based camera for medical imaging," Nucl. Instrum. Methods Phys. Res. A, vol. A513, no. $1-2$, pp. 23-26, 2003. 
[8] F. J. Beekman and B. Vastenhouw, "Design and simulation of a highresolution stationary SPECT system for small animals," Phys. Med. Biol., vol. 49, no. 19, pp. 4579-4592, 2004.

[9] E. Miyata, M. Miki, N. Tawa, D. Kamiyama, and K. Miyaguchi, "Development of new X-ray imaging device sensitive to $0.1-100 \mathrm{keV}$," Nucl. Instrum. Methods Phys. Res. A, vol. A525, pp. 122-125, 2004.

[10] G. A. de Vree, A. H. Westra, I. Moody, F. van der Have, C. M. Ligtvoet, and F. J. Beekman, "Photon-counting gamma camera based on an electron-multiplying CCD," IEEE Trans. Nucl. Sci., vol. 52, no. 3, pp. 580-588, Jun. 2005.

[11] J. Kataoka, T. Saito, Y. Kuramoto, T. Ikagawa, Y. Yatsu, J. Kotoku, M. Arimoto, N. Kawai, Y. Ishikawa, and N. Kawabata, "Recent progress of avalanche photodiodes in high-resolution X-rays and g-rays detection," Nucl. Instrum. Methods Phys. Res. A, vol. A541, pp. 398-404, 2005.

[12] F. J. Beekman and G. A. de Vree, "Photon-counting versus an integrating CCD-based gamma camera: important consequences for spatial resolution," Phys. Med. Biol., vol. 50, no. 12, pp. N109-N119, 2005.

[13] C. Ponchut, F. Zontone, and H. Graafsma, "Experimental comparison of pixel detector arrays and CCD-based systems for X-ray area detection on synchrotron beamlines," IEEE Trans. Nucl. Sci., vol. 52, no. 5, pp. 1760-1765, Oct. 2005.

[14] V. V. Nagarkar, I. Shestakova, V. Gaysinskiy, S. V. Tipnis, B. Singh, W. Barber, B. Hasegawa, and G. Entine, "A CCD-based detector for SPECT," IEEE Trans. Nucl. Sci., vol. 53, no. 1, pp. 54-58, Feb. 2006.

[15] L. J. Meng, "An intensified EMCCD camera for low energy gamma ray imaging applications," IEEE Trans. Nucl. Sci., vol. 53, no. 4, pp. 2376-2384, Aug. 2006.

[16] H. H. Barrett and W. Swindell, Radiological Imaging. The Theory of Image Formation, Detection and Processing. New York: Academic, 1981.

[17] F. J. Beekman, F. van der Have, B. Vastenhouw, A. J. A. van der Linden, P. R. van Rijk, J. P. H. Burbach, and M. P. Schmidt, "U-SPECT-I: A novel system for submillimeter-resolution tomography with radiolabeled molecules in mice," J. Nucl. Med., vol. 46, no. 7, pp. 1194-1200, 2005.

[18] F. J. Beekman and F. van der Have, "The Pinhole: Gateway to ultrahigh resolution three-dimensional radionuclide imaging," Eur. J. Nucl. Med. Mol. Imag., vol. 34, no. 2, pp. 151-161, 2007.
[19] J. W. T. Heemskerk, A. H. Westra, P. M. Linotte, C. M. Ligtvoet, W Zbijewski, and F. J. Beekman, "Front-illuminated versus back-illuminated photon-counting CCD-based gamma camera: Important consequences for spatial resolution and energy resolution," Phys. Med. Biol., vol. 52, no. 8, pp. N149-N162, 2007.

[20] J. Hynecek and N. Nishiwaki, "Excess noise and other important characteristics of low light level imaging using charge multiplying CCDs," IEEE Trans. Electron Devices, vol. 50, no. 1, pp. 239-245, 2003.

[21] T. Plakhotnik, A. Chennu, and A. V. Zvyagin, "Statistics of singleelectron signals in electron-multiplying charge-coupled devices," IEEE Trans. Electron Devices, vol. 53, no. 4, pp. 618-622, 2006.

[22] Dark Signal and Clock-Induced Charge in L3Vision CCD Sensors, E2V low-light technical note 4 [Online]. Available: http://www.e2v.com/products/CCD-and-cmos-imaging-and-semiconductors/imaging-13vision/datasheets-and-technical-papers.cfm

[23] P. Jerram, P. Pool, R. Bell, D. Burt, S. Bowring, S. Spencer, M. Hazelwood, I. Moody, N. Catlett, and P. Heyes, "The LLLCCD: Low light imaging without the need for an intensifier," Proc. SPIE., vol. 4306, pp. 178-186, 2001

[24] J. Hynecek, "Impactron-A new solid state image intensifier," IEEE Trans. Electron Devices, vol. 48, pp. 2238-2241, 2001.

[25] M. S. Robbins and B. J. Hadwen, "The noise performance of electron multiplying charge-coupled devices," IEEE Trans. Electron Devices, vol. 50, pp. 1227-1232, 2003.

[26] [Online]. Available: http://www.e2v.com/products/advancedimaging-and-semiconductors/imaging-13vision/datasheets-and-technical-papers.cfm

[27] C. D. Mackay, "High-speed digital CCD cameras-principals and applications," in Fluorescent and Luminescent Probes for Biological Activity, 2nd ed. New York: Academic, 1999, pp. 517-524.

[28] B. W. Miller, H. B. Bradford, H. H. Barrett, I. Shestakova, B. Singh, and V. V. Nagarkar, "Single-photon spatial and energy resolution enhancement of a columnar CsI(Tl)/EMCCD gamma-camera using maximum-likelihood estimation," in Proc. SPIE, 2006, 6142 61421T.

[29] F. van der Have and F. J. Beekman, "Characterization of photon penetration and scatter in micro-pinholes," Phys. Med. Biol., vol. 49, no. 8, pp. 1369-86, 2004. 